\title{
Effects of Dietary Supplementation with Myo-inositol on Hepatic Expression of Glycolytic and Fructolytic Enzyme Genes in Rats Fed a High-sucrose Diet
}

\author{
Mayu Hibi ${ }^{1}$, Sakura Sugiura ${ }^{2}$, Tomoyuki Nakagawa ${ }^{1,2}$, Takashi Hayakawa ${ }^{1,2}$, and \\ Masaya Shimada ${ }^{1,2 *}$ \\ ${ }^{1}$ Division of Life Science for Food, Department of Life Science and Chemistry, Graduate School of Natural Science and Technology, Gifu \\ University, 1-1 Yanagido, Gifu-shi, Gifu 501-1193, JAPAN \\ ${ }^{2}$ Department of Applied Life Science, Faculty of Applied Biological Sciences, Gifu University, 1-1 Yanagido, Gifu-shi, Gifu 501-1193, JAPAN
}

\begin{abstract}
We examined effects of a major lipotrope, myo-inositol, on the expression of primary glycolytic (glucokinase and phosphofructokinase) and fructolytic enzyme (ketohexokinase [KHK] and aldolase B) genes in the livers of rats fed a control diet, high-sucrose diet, or high-sucrose diet supplemented with $0.5 \%$ myo-inositol for $14 \mathrm{~d}$. Supplementation with myo-inositol decreased the hepatic expression of fructolytic enzyme genes, but not that of glycolytic enzyme genes, and the levels of triglycerides, fatty acid synthase, and KHK proteins in high-sucrose diet-induced fatty liver. The study results suggest that myo-inositol represses primary fructlysis, but not glycolysis, in high-sucrose diet-induced fatty liver.
\end{abstract}

Key words: KHK, liver, myo-inositol, rats, sucrose

\section{Introduction}

Sucrose is digested by sucrase and metabolized into monosaccharides, glucose and fructose, in the small intestine. The two monosaccharides, after absorption from the small intestine, reach the liver via the portal vein. In the liver, glucose can be partially converted into fatty acids and stored as triglycerides after it is metabolized by two highly regulated enzymes in glycolysis, glucokinase (GK) and phosphofructokinase (PFK). In contrast, fructose is metabolized by two key fructolytic enzymes, ketohexokinase (KHK) and aldolase B (ALDOB) and is more rapidly utilized than glucose for de novo lipogenesis because fructose bypasses the glycolytic rate-limiting step, PFK. Thus, excessive intake of sucrose promotes not only the prevalence and/or development of fatty liver, but also that of other metabolic diseases, such as insulin resistance and type 2 diabetes ${ }^{1)}$. Therefore, repression of sucrose-induced fatty liver is a key strategy for the prevention of these metabolic diseases.

Myo-inositol, the most common form of inositol of the nine distinct stereoisomers, is well-known to be a major lipotrope. It is found in the free form in fruits such as kiwi or citrus $^{2}$. Several animal studies have reported that dietary supplementation with myo-inositol effectively reduces the accumulation of triglycerides, expression of genes of fatty acid synthesis enzymes, and activity of fatty acid synthesis enzymes in rats with high-sucrose diet-induced fatty liver $^{3-7)}$. In addition, we have shown that dietary supplementation with myo-inositol decreases not only the accumulation of triglycerides and expression of fatty acid synthesis enzyme genes and protein, but also the expression of fructolysis enzyme genes in the fatty liver of rats fed a high-fructose diet $^{8)}$. However, it remains unclear whether the intake of myo-inositol affects the expression of genes related to either glycolysis or fructolysis, or both, in high-sucrose-induced fatty liver.

In this study, we examined the effects of dietary supplementation with myo-inositol on the hepatic expression of primary glycolytic and fructolytic enzyme genes in the livers of rats fed a high-sucrose diet.

\section{Experimental}

\subsection{Animals and diets}

Four-week-old Wistar male rats (SLC, Shizuoka, Japan) were housed individually in metal cages in a temperaturecontrolled room $\left(22^{\circ} \mathrm{C} \pm 2^{\circ} \mathrm{C}\right)$ under a 12 -h light/dark cycle

\footnotetext{
*Correspondence to: Masaya Shimada, Department of Applied Life Science, Faculty of Applied Biological Sciences, Gifu University, 1-1 Yanagido, Gifu-shi, Gifu 501-1193, JAPAN

E-mail: mshimada@gifu-u.ac.jp

Accepted January 26, 2021 (received for review November 29, 2020)
}

Journal of Oleo Science ISSN 1345-8957 print / ISSN 1347-3352 online

http://www.jstage.jst.go.jp/browse/jos/ http://mc.manusriptcentral.com/jjocs 
(lights on from 07:00-19:00) and fed a laboratory chow diet (MF; Oriental Yeast, Tokyo, Japan). After an acclimation period of $5 \mathrm{~d}$, the rats were assigned to one of the following 3 diet groups $(\mathrm{n}=6)$ for $14 \mathrm{~d}$ : control diet, high-sucrose diet, or high-sucrose diet supplemented with $0.5 \%$ myoinositol. The purity of myo-inositol (Nacalai Tesque, Kyoto, Japan)was $\geq 99.0 \%$. Details of diet composition are presented in Table 1. The animals had free access to food and water. At the end of the feeding period, the rats were euthanized by decapitation under isoflurane anesthesia after a 4-h fasting period. Liver tissues were harvested and stored in liquid nitrogen. All animal care and experimental procedures were approved by the Gifu University Animal Care and Usage Committee.

\subsection{Serum lipid assay}

Blood samples were collected after decapitation and centrifuged at $1,710 \times \mathrm{g}$ for $15 \mathrm{~min}$ at $4^{\circ} \mathrm{C}$. Serum samples were collected and stored at $-30^{\circ} \mathrm{C}$ until use. The concentrations of glucose, insulin, triglycerides, total cholesterol, and phospholipids in the samples were measured using commercial kits (Glucose CII-test Wako, LBIS Insulin-Rat [U-E type], Triglyceride E-test Wako, Cholesterol E-test Wako, and Phospholipid C-test Wako, respectively; FUJIFILM Wako Pure Chemical Corporation, Osaka, Japan).

\subsection{Hepatic lipid assay}

Total lipids from the rat liver tissues were extracted using the method described by Bligh and Dyer ${ }^{9)}$ with some modifications. Briefly, frozen liver tissue was homogenized and mixed with chloroform, methanol, and $0.1 \mathrm{M} \mathrm{KCl}$ in a ratio of 1:1:1 $(\mathrm{v} / \mathrm{v} / \mathrm{v})$. The homogenates were centrifuged at
$800 \times \mathrm{g}$ for $10 \mathrm{~min}$ at room temperature and the lower organic phases were collected and dried as lipid extracts. The lipid extract samples were dissolved in isopropanol, and the amounts of triglycerides and cholesterol in the samples were measured using commercial kits (Triglyceride E-test Wako and Cholesterol E-test Wako, respectively; FUJIFILM Wako Pure Chemical Corporation).

\subsection{Real-time quantitative PCR}

Total RNA was extracted from the frozen liver tissues using a RNeasy Mini kit, (Qiagen, Tokyo, Japan)according to the manufacturer's instructions and stored at $-80^{\circ} \mathrm{C}$ before use. Total RNA was converted to cDNA using a ReverTra Ace qPCR RT kit(Toyobo, Osaka, Japan)according to the manufacturer's instructions. The cDNA samples were stored at $-80^{\circ} \mathrm{C}$ until they were ready for use. Realtime quantitative PCR was performed in a final volume of $20 \mu \mathrm{L}$ containing SYBR Green(TB Green Premix Ex Taq; Takara, Shiga, Japan), $0.2 \mu \mathrm{M}$ of each primer, Rox reference dye, and 20 ng of cDNA, using a real-time PCR system (Step One Plus; Applied Biosystems Japan, Tokyo, Japan). The primers used are listed in Table 2. Expression of each tested gene was normalized to the housekeeping gene 36B4 using the $2^{-\Delta \Delta C \mathrm{~T}}$ method $^{10)}$.

\subsection{Immunoblot}

The frozen liver tissues were homogenized in ice-cold RIPA buffer (FUJIFILM Wako Pure Chemical Corporation) containing protease inhibitors (Complete Mini; Sigma-Aldrich Japan, Tokyo, Japan). The homogenates were centrifuged at $16,100 \times \mathrm{g}$ for $30 \mathrm{~min}$ at $4^{\circ} \mathrm{C}$ and the lysate samples collected. Protein concentrations in the samples were de-

Table 1 Composition of diets.

\begin{tabular}{lccc}
\hline & Control & Sucrose & $\begin{array}{c}\text { Sucrose } \\
\text { +myo-inositol }\end{array}$ \\
\cline { 2 - 4 } & & $\%$ & \\
\hline Casein & 20.0 & 20.0 & 20.0 \\
C-Cornstarch & 64.9 & - & - \\
Sucrose & - & 64.9 & 64.4 \\
Corn oil & 5.0 & 5.0 & 5.0 \\
Cellulose & 5.0 & 5.0 & 5.0 \\
AIN 93G mineral mixture & 3.5 & 3.5 & 3.5 \\
AIN 93 vitamin mixture & 1.0 & 1.0 & 1.0 \\
L-cystine & 0.3 & 0.3 & 0.3 \\
Choline bitartrate & 0.3 & 0.3 & 0.3 \\
Myo-inositol & - & - & 0.5 \\
Total & 100 & 100 & 100 \\
\hline
\end{tabular}

Control, control diet; sucrose, high-sucrose diet; sucrose+myo-inositol, high-sucrose diet plus $0.5 \%$ myo-inositol. 
Table 2 Sequences of primers.

\begin{tabular}{lll}
\hline \multicolumn{2}{c}{ Forward } & \multicolumn{1}{c}{ Reverse } \\
\hline GK & 5'- AATGTGAGGTCGGCATGATT -3' & 5'- CACATTCTGCATTTCCTCCA -3' \\
PFK & 5'- TTGGTGATCGGTGGCTTT -3' & 5'- TGACGCACATGACGATACAG -3' \\
KHK & 5'- CACCTTCAATGCCTCTGTCA -3' & 5'- CCTGCAAGCCACACTTCTT -3' \\
ALDOB & 5'- TTGCCAATGGGAAGGGTA -3' & 5'- ATCCTCTGTAGGCGGTTTCC -3' \\
36 B4 & 5'- CGAGAAGACCTCTTTCTTCCAA -3' & 5'- AGTCTTTATCAGCTGCACATCG -3' \\
\hline
\end{tabular}

Table 3 Physiological and biochemical parameters in rats fed a control diet, high-sucrose diet, or high-sucrose diet supplemented with $0.5 \%$ myo-inositol for $14 \mathrm{~d}$.

\begin{tabular}{lccc}
\hline & Control & Sucrose & Sucrose+MI \\
\hline Final body weight $(\mathrm{g})$ & $169 \pm 2$ & $171 \pm 4$ & $163 \pm 1.2$ \\
Food intake $(\mathrm{g} / \mathrm{d})$ & $14.7 \pm 0.3$ & $14.7 \pm 0.5$ & $14.8 \pm 0.2$ \\
Serum & & & \\
$\quad$ Glucose $(\mathrm{mg} / 100 \mathrm{~mL})$ & $134 \pm 4$ & $140 \pm 3$ & $147 \pm 5$ \\
$\quad$ Insulin $(\mathrm{ng} / \mathrm{mL})$ & $0.742 \pm 0.220$ & $0.726 \pm 0.149$ & $0.640 \pm 0.094$ \\
$\quad$ Triglycerides $(\mathrm{mg} / 100 \mathrm{~mL})$ & $115 \pm 8^{\mathrm{b}}$ & $270 \pm 23^{\mathrm{a}}$ & $229 \pm 40^{\mathrm{a}}$ \\
$\quad$ Total cholesterol $(\mathrm{mg} / 100 \mathrm{~mL})$ & $85 \pm 4$ & $97 \pm 5$ & $101 \pm 5$ \\
$\quad$ Phospholipids $(\mathrm{mg} / 100 \mathrm{~mL})$ & $170 \pm 8^{\mathrm{b}}$ & $200 \pm 8^{\mathrm{ab}}$ & $213 \pm 8^{\mathrm{a}}$ \\
Mesenteric adipose tissue $(\mathrm{g} / 100 \mathrm{~g}$ body weight) & $0.589 \pm 0.017$ & $0.596 \pm 0.046$ & $0.605 \pm 0.027$ \\
Liver & & & \\
$\quad$ Weight $(\mathrm{g} / 100 \mathrm{~g}$ body weight) & $4.65 \pm 0.08^{\mathrm{b}}$ & $5.79 \pm 0.19^{\mathrm{a}}$ & $5.38 \pm 0.21^{\mathrm{a}}$ \\
Triglyceride $(\mathrm{mg} / \mathrm{g}$ liver $)$ & $38.4 \pm 5.7^{\mathrm{b}}$ & $117 \pm 13^{\mathrm{a}}$ & $37.6 \pm 5.2^{\mathrm{b}}$ \\
Cholesterol $(\mathrm{mg} / \mathrm{g}$ liver) & $15.6 \pm 0.5^{\mathrm{a}}$ & $16.6 \pm 0.9^{\mathrm{a}}$ & $13.0 \pm 0.4^{\mathrm{b}}$ \\
\hline
\end{tabular}

Values are expressed as mean \pm standard error of mean $(n=6)$. Values in a column that do not share a common superscript are significantly different ( $p<0.05$, Tukey's test based on one-way analysis of variance). Control, control diet; sucrose, high-sucrose diet; sucrose+myo-inositol, high-sucrose diet plus $0.5 \%$ myo-inositol.

termined using the BCA assay (BCA protein assay kit; Takara) and stored at $-80^{\circ} \mathrm{C}$ until ready for use. The lysate samples containing an equal amount of protein were separated by $5 \%$ and $12.5 \%$ SDS-PAGE and then transferred onto PVDF membranes $(0.2-\mu \mathrm{m}$, hydrophobic, Clear Trans SP PVDF Membrane; FUJIFILM Wako Pure Chemical Corporation). After transfer, the membranes were washed in PBS with $0.1 \%$ Tween 20 (PBS-T) and blocked in 3-5\% skimmed milk in PBS-T for $1 \mathrm{~h}$ at room temperature. Thereafter, the membranes were incubated overnight at $4^{\circ} \mathrm{C}$ with the following primary antibodies: anti-fatty acid synthase (FAS) antibody (Gene Tex, CA, USA), anti-KHK (Gene Tex) antibody, and anti- $\alpha$-tubulin antibody (Cell Signaling Technology Japan, Tokyo, Japan). Subsequently, the membranes were washed in PBS-T and incubated for $1 \mathrm{~h}$ at $4^{\circ} \mathrm{C}$ with the secondary antibody conjugated to HRP (Cell Signaling Technology Japan). After washing with PBS-T, the signals were detected by ECL (Immnostar LD; FUJIFILM Wako Pure Chemical Corporation)according to the manufacturer's instructions and using an imaging system (LAS3000; FUJIFILM, Tokyo, Japan). The expression of each protein was normalized using a loading control, $\alpha$-tubulin.

\subsection{Statistical analysis}

Values are expressed as mean \pm SEM. The significance of differences between the groups was determined using Tukey's test, based on one-way analysis of variance. $P<$ 0.05 indicated statistical significance.

\section{Results and Discussion}

In this study, serum levels of glucose, insulin, and total cholesterol did not significantly differ between rats fed a control diet, high-sucrose diet, and high-sucrose diet supplemented with myo-inositol. Serum triglyceride levels were significantly higher in the high-sucrose groups 
(A)

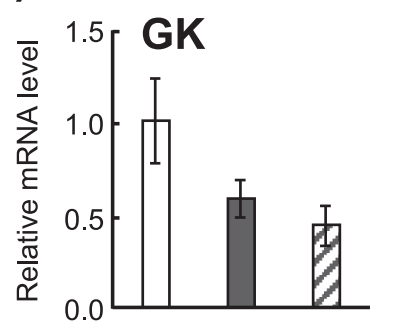

(B)
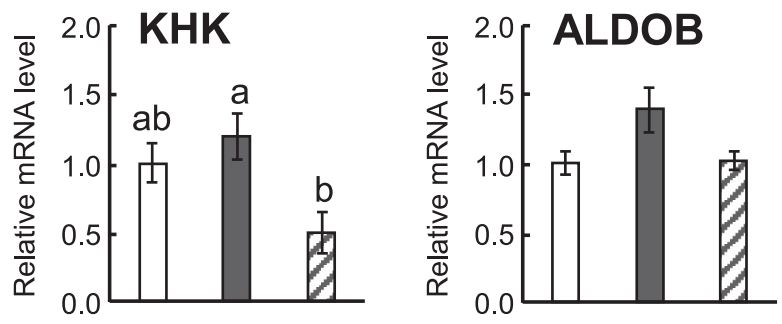

$\square$ Control $\square$ Sucrose $\square$ Sucrose+myo-inositol

Fig. 1 Effects of dietary supplementation with myoinositol on hepatic expression of glycolytic and fructolytic enzyme genes in rats fed a high-sucrose diet.

Hepatic mRNA levels of(A) glycolytic and (B) fructolytic enzyme genes were analyzed by realtime quantitative PCR. The mRNA levels of the tested genes were normalized to that of 36B4. Values are expressed as mean $\pm \operatorname{SEM}(n=6)$. Values not sharing a common superscript are significantly different $(p<0.05$, Tukey's test based on one-way analysis of variance). Control, control diet; sucrose, high-sucrose diet; sucrose+myoinositol, high-sucrose diet plus $0.5 \%$ myo-inositol.

without/with myo-inositol supplementation than in the control group. Serum phospholipid levels tended to be higher in the high-sucrose group and were significantly higher in the high-sucrose plus myo-inositol group than in the control group. In contrast to the biochemical lipid parameters, dietary supplementation with myo-inositol remarkably decreased hepatic levels of triglycerides and cholesterol in rats fed a high-sucrose $\operatorname{diet}($ Table 3 ). In addition, myo-inositol supplementation reduced the highsucrose diet-induced hepatic expression of FAS protein (Fig. 2B). Considering that dietary supplementation with myo-inositol lowered the levels of hepatic lipids, it is likely that myo-inositol decreases triglyceride accumulation via repressed fatty acid synthesis in the liver. However, it should be noted that myo-inositol supplementation did not decrease serum triglyceride levels. In this study, supplementation with myo-inositol increased the levels of serum phospholipids, which suggests the enhanced secretion of

(A)

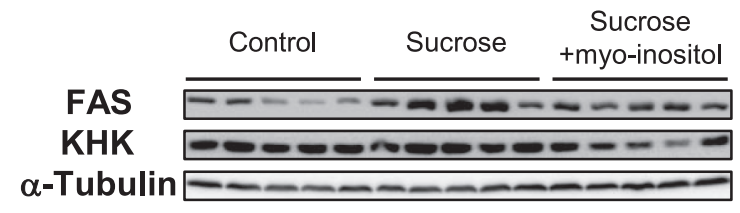

(B)

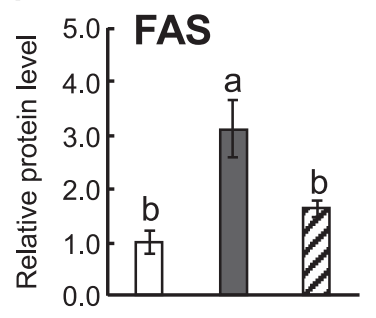

(C)

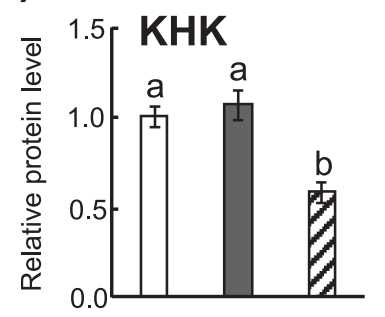

$\square$ Control $\square$ Sucrose $\square$ Sucrose+myo-inositol

Fig. 2 Effects of dietary supplementation with myoinositol on hepatic expression of FAS and KHK proteins.

(A) Protein expression was measured in liver tissue lysates by immunoblot. Quantitative analysis of (B) FAS and (C) KHK protein based on densitometry of immunoblot. The protein levels of the tested protein were normalized to that of $\alpha$-tubulin. Values are expressed as means $\pm \operatorname{SEM}(n=5)$. Values not sharing a common superscript are significantly different $(p<0.05$, Tukey's test based on one-way analysis of variance). Control, control diet; Sucrose, high-sucrose diet; Sucrose+myoinositol, high-sucrose diet plus $0.5 \%$ myo-inositol.

very-low-density lipoprotein from the liver, but not the enhanced secretion of chylomicron from the small intestine. Thus, this may explain why myo-inositol did not decrease serum triglyceride levels.

We showed that dietary supplementation with myo-inositol significantly lowered the expression of the KHK gene and KHK protein $(p<0.05)$ and tended to lower the expression of ALDOB, $p=0.068$, (Figs. $1 \mathrm{~B}$ and $2 \mathrm{C}$ ). To the best of our knowledge, this is the first study to report that myoinositol supplementation remarkably decreases the expression of the KHK gene and KHK protein as well as the accumulation of triglycerides and expression of the FAS protein in high-sucrose diet-induced fatty liver in rats. Our previous study reported that dietary supplementation with myoinositol reduced the expression of fructolytic and fatty acid synthesis enzyme genes and accumulation of triglycerides in the fatty livers of rats fed a high-fructose diet ${ }^{8}$. Therefore, it is most likely that myo-inositol is a major lipotrope that lowers triglyceride accumulation via repressed fructolysis and fatty acid synthesis in fatty liver induced by high- 
sucrose diets as well as high-fructose diets.

However, the underlying mechanisms by which myo-inositol decreases the expression of KHK and ALDOB genes remains unknown. Several studies have reported that a key lipogenic transcription factor, carbohydrate-responsive element-binding protein (ChREBP), binds to the KHK gene promoter in human hepatocytes, HepG2 cells ${ }^{11)}$, and that liver-specific ChREBP-null rats repress high fructose-induced expression of KHK and ALDOB genes ${ }^{12)}$. ChREBP is translocated into the nucleus and activated by dephosphorylation at its sites, serine 196 and threonine 666 via protein phosphatase $2 \mathrm{~A}$, which is activated in the pentosephosphate pathway ${ }^{13)}$. Several studies have shown that administration of a high-sucrose diet supplemented with myo-inositol in rats lowers hepatic activities of glucose6 -phosphate dehydrogenase ${ }^{4-6)}$, which catalyzes a ratelimiting step of the pentose-phosphate pathway and produces NADPH for fatty acid synthesis, indicating that myoinositol could repress the dephosphorylation and activation of ChREBP. In addition, we reported that treatment with myo-inositol attenuated high fructose-induced binding of ChREBP to the FAS promoter in the rat liver ${ }^{14)}$. Moreover, in vitro studies have shown that inositol phosphates, which are secondary messengers containing myo-inositol as their component, activate histone deacetylases (HDACs), which are transcriptional repressors ${ }^{15,16)}$. Therefore, a decrease in the expression of KHK and ALDOB genes by myo-inositol may be involved in the synergistic inactivation of ChREBP and activation of HDACs. However, this hypothesis requires further investigation.

In contrast to the decreased expression of the fructolytic enzyme genes by myo-inositol, myo-inositol did not affect the expression of GK and PFK genes (Fig. 1A). Glucose can be taken up by the liver and converted into fatty acids, especially under conditions of excess energy, such as obesity. In this study, feeding rats a high-sucrose diet with or without myo-inositol for $14 \mathrm{~d}$ did not significantly increase mesenteric adipose tissue compared with that in rats fed a control diet (Table 3 ). This may explain why myo-inositol did not affect the expression of GK and PFK genes. Therefore, further studies should investigate whether intake of myo-inositol decreases the expression of glycolytic enzyme genes in the liver of obese rats fed a high-fat/high-sucrose diet for longer durations.

It should be noted that myo-inositol supplementation reduced the expression of KHK more potently than it did that of ALDOB. Several studies have demonstrated that KHK-knockout mice exhibit improved fatty liver induced by high-fat and high-fat/high-sucrose diets ${ }^{17,18)}$. In addition, Lanaspa et al. showed that KHK/ALDOB-double knockout mice, compared with ALDOB-knockout mice, repressed hepatic inflammation and fibrosis when they were exposed to fructose ${ }^{19)}$. Therefore, our data that myo-inositol reduced the expression of KHK prior to that of ALDOB may be useful for the prevention of high-sucrose diet-induced fatty liver and other metabolic diseases, such as insulin resistance and type 2 diabetes.

\section{Conclusions}

We showed that dietary supplementation with myo-inositol decreases hepatic triglyceride levels and the expression of fructolytic enzyme (KHK and ALDOB) genes and KHK proteins, but not that of glycolytic enzyme (GK and PFK) genes in rats fed a high-sucrose diet. The study results suggest that myo-inositol represses primary fructlysis, but not glycolysis, in high-sucrose diet-induced fatty liver.

\section{Acknowledgments}

This work was supported by JSPS KAKENHI Grant number JP18K02237.

\section{References}

1) Stanhope, K.L. Sugar consumption, metabolic disease and obesity: The state of the controversy. Crit. Rev. Clin. Lab. Sci. 53, 52-67(2016).

2) Fardet, A.; Chardigny, J.M. Plant-based foods as a source of lipotropes for human nutrition: a survey of in vivo studies. Crit. Rev. Food. Sci. Nutr. 53, 535590 (2013).

3) Beach, D.C.; Flick, P.K. Early effect of myo-inositol deficiency on fatty acid synthetic enzymes of rat liver. Biochim. Biophys. Acta 711, 452-459(1982).

4) Katayama, T. Effect of dietary addition of myo-inositol on lipid metabolism in rats fed sucrose or corn starch. Nutr. Res. 14, 699-706 (1994).

5) Katayama, T. Effects of dietary myo-inositol or phytic acid on hepatic concentrations of lipids and hepatic activities of lipogenic enzymes in rats fed on corn starch or sucrose. Nutr. Res. 17, 721-728(1997).

6) Onomi, S.; Katayama, T.; Sato, K. Effects of dietary myo-inositol related compounds on sucrose-mediated hepatic lipid accumulation in rats. Nutr. Res. 19, 14011409 (1999).

7) Okazaki, Y.; Sekita, A.; Katayama, T. Intake of phytic acid and myo-inositol lowers hepatic lipogenic gene expression and modulates gut microbiota in rats fed a high-sucrose diet. Biomed. Rep. 8, 466-474(2018).

8) Shimada, M.; Hibino, M.; Takeshita, A. Dietary supplementation with myo-inositol reduces hepatic triglyceride accumulation and expression of both fructolytic and lipogenic genes in rats fed a high-fructose diet. 
Nutr Res 47, 21-27 (2017).

9) Bligh, E.G.; Dyer, W.J. A rapid method of total lipid extraction and purification. Can. J. Biochem. Physiol. 37, 911-917 (1959).

10) Livak, K.J.; Schmittgen, T.D. Analysis of relative gene expression data using real-time quantitative PCR and the 2 (-Delta Delta C (T) ) method. Methods 25, 402408(2001).

11) Lanaspa, M.A.; Sanchez-Lozada, L.G.; Cicerchi, C.; Li, N.; Roncal-Jimenez, C.A.; Ishimoto, T.; Le, M.; Garcia, G.E.; Thomas, J.B.; Rivard, C.J.; Andres-Hernando, A.; Hunter, B.; Schreiner, G.; Rodriguez-Iturbe, B.; Sautin, Y.Y.; Johnson, R.J. Uric acid stimulates fructokinase and accelerates fructose metabolism in the development of fatty liver. PLoS One 7, e47948 (2012).

12) Erion, D.M.; Popov, V.; Hsiao, J.J.; Vatner, D.; Mitchell, K.; Yonemitsu, S.; Nagai, Y.; Kahn, M.; Gillum, M.P.; Dong, J.; Murray, S.F.; Manchem, V.P.; Bhanot, S.; Cline, G.W.; Shulman, G.I.; Samuel, V.T. The role of the carbohydrate response element-binding protein in male fructose-fed rats. Endocrinology 154, 36-44 (2013).

13) Uyeda, K.; Repa, J.J. Carbohydrate response element binding protein ChREBP, a transcription factor coupling hepatic glucose utilization and lipid synthesis. Cell Metab. 2, 107-110 (2006).

14) Shimada, M.; Ichigo, Y.; Shirouchi, B.; Takashima, S.; Inagaki, M.; Nakagawa, T.; Hayakawa, T. Treatment with myo-inositol attenuates binding of the carbohydrate-responsive element-binding protein to the ChREBP- $\beta$ and FASN genes in rat nonalcoholic fatty liver induced by high-fructose diet. Nutr. Res. 64,
49-55 (2019).

15) Millard, C.J.; Watson, P.J.; Celardo, I.; Gordiyenko, Y.; Cowley, S.M.; Robinson, C.V.; Fairall, L.; Schwabe, J.W. Class I HDACs share a common mechanism of regulation by inositol phosphates. Mol. Cell 51, 57-67 (2013) .

16) Watson, P.J.; Millard, C.J.; Riley, A.M.; Robertson, N.S.; Wright, L.C.; Godage, H.Y.; Cowley, S.M.; Jamieson, A.G.; Potter, B.V.; Schwabe, J.W. Insights into the activation mechanism of class I HDAC complexes by inositol phosphates. Nat. Commun. 7, 11262 (2016).

17) Ishimoto, T.; Lanaspa, M.A.; Rivard, C.J.; RoncalJimenez, C.A.; Orlicky, D.J.; Cicerchi, C.; McMahan, R.H.; Abdelmalek, M.F.; Rosen, H.R.; Jackman, M.R.; MacLean, P.S.; Diggle, C.P.; Asipu, A.; Inaba, S.; Kosugi, T.; Sato, W.; Maruyama, S.; Sánchez-Lozada, L.G.; Sautin, Y.Y.; Hill, J.O.; Bonthron, D.T.; Johnson, R.J. High-fat and high-sucrose (western) diet induces steatohepatitis that is dependent on fructokinase. Hepatology 58, 1632-1643(2013).

18) Miller, C.O.; Yang, X.; Lu, K.; Cao, J.; Herath, K.; Rosahl, T.W.; Askew, R.; Pavlovic, G.; Zhou, G.; Li, C.; Akiyama, T.E. Ketohexokinase knockout mice, a model for essential fructosuria, exhibit altered fructose metabolism and are protected from diet-induced metabolic defects. Am. J. Physiol. Endocrinol. Metab. 315, E386-E393 (2018).

19) Lanaspa MA, Andres-Hernando A, Orlicky DJ, Cicerchi C, Jang C, Li N, Milagres T, Kuwabara M, Wempe MF, Rabinowitz JD, Johnson RJ, Tolan DR. Ketohexokinase C blockade ameliorates fructose-induced metabolic dysfunction in fructose-sensitive mice. J. Clin. Invest. 128, 2226-2238(2018). 